\title{
Downregulation of NLRP2 inhibits HUVEC viability by inhibiting the MAPK signaling pathway
}

\author{
XIAOLU ZHANG ${ }^{1 *}$, XINLEI LU ${ }^{2 *}$, LIMEI YU ${ }^{1}$, YUFENG GU ${ }^{1}$ and FUZHENG QU ${ }^{3}$ \\ ${ }^{1}$ Department of Laboratory Medicine, Yuhuangding Hospital of Qingdao University, Yantai, Shandong 264000; \\ ${ }^{2}$ Department of Cardiology, Weihai Central Hospital, Weihai, Shandong 264200; ${ }^{3}$ Department of Cardiology, \\ Yantai Affiliated Hospital of Binzhou Medical University, Yantai, Shandong 264100, P.R. China
}

Received January 16, 2018; Accepted September 14, 2018

DOI: $10.3892 / \mathrm{mmr} .2018 .9625$

\begin{abstract}
Nucleotide-binding oligomerization domain (NOD)-like receptor proteins (NLRPs) are a subfamily of NOD-like receptors (NLRs) that mainly participate in innate immunity. Among the 14 NLRPs, studies on NLRP2 are few and mostly focus on its functions in reproduction and embryonic development. To the best of the authors' knowledge, there has been no research on the function of NLRP2 in human umbilical vein endothelial cells (HUVECs). The present study knockdown the expression of NLRP2 by transfecting a short interfering (si)RNA (siNLRP2) into HUVECs and investigating its effects on HUVECs. It was identified using a Cell Counting kit- 8 assay that knockdown of NLRP2 can inhibit cell proliferation in HUVECs. The results of wound healing and Transwell assays indicated that migration and invasion were also suppressed by siNLRP2 transfection in HUVECs. Flow cytometry demonstrated that siNLRP2 induced cell cycle arrest and apoptosis in HUVECs. Western blot analysis revealed that the expression levels of cell cycle and apoptosis-associated proteins were markedly changed. In addition, knockdown of NLRP 2 inhibited the mitogen-activated protein kinase (MAPK) signaling pathway by elevating extracellular signal-regulated kinase phosphorylation levels and reducing proto-oncogene serine/threonine-protein kinase expression. Taken together, it was concluded that NLRP2 served an important role in maintaining cell viability, proliferation and motility in HUVECs, mainly by promoting the MAPK signaling pathway.
\end{abstract}

Correspondence to: Dr Fuzheng Qu, Department of Cardiology, Yantai Affiliated Hospital of Binzhou Medical University, 717 Jinbu Street, Muping, Yantai, Shandong 264100, P.R. China E-mail: qufuzheng2018@163.com

*Contributed equally

Key words: nucleotide-binding oligomerization domain-like receptor protein 2, human umbilical vein endothelial cells, cell proliferation, apoptosis, mitogen-activated protein kinase signaling pathway

\section{Introduction}

Nucleotide-binding oligomerization domain-like receptors (NLRs) are a class of cytoplasmic pattern-recognition receptors involved in identifying harmful substances in the cytoplasm and forming inflammasomes to transform these antigens into immune-initiating signals $(1,2)$. There are four subfamilies in NLRs, including NLRA, NLRB, NLRC and NLRP (3). The NLRP family (NLRPs) is distinguished by a pyrin domain in the N-terminal. Among the 22 known NLRs, the NLRP family contains 14 members, NLRP1-14 (4). The current understanding of NLRPs is concentrated on NLRP1, NLRP3 and NLRP6. When stimulated by a cytoplasmic antigen signal, these proteins recruit the adaptor protein ASC by the pyrin domain to form a multi-protein complex, which further activates caspase-1 and cleaves effector pro-inflammatory cytokines interleukin (IL)-1b and IL-18 (5-7). However, the studies of NLRP2 are limited and the majority of them focus on its functions in reproduction and embryonic development. For example, Tilburgs et al (8) identified that NLRP2 was involved in preventing unwanted antifetal responses by suppressing nuclear factor $\kappa \mathrm{B}$ signaling and major histocompatibility complex, class I, C expression in human trophoblasts. Mahadevan et al (9) identified that maternally expressed NLRP2 links the subcortical maternal complex to fertility, embryogenesis and epigenetic reprogramming. Peng et al (10) identified that NLRP2 served an important role in early embryonic development in mice. However, NLRP2, as a systemic protein associated with biological reproduction and embryonic development, must serve an important role in a variety of biological processes, although relevant studies are lacking.

Vascular endothelial cells, located between the bloodstream and tissues, are involved in numerous physiological and pathological processes, including (tumor) angiogenesis, inflammation and wound healing $(11,12)$. A recent study revealed that NLRP2 was significantly upregulated in a mouse model of ischemic stroke and served important roles in the pathophysiological processes (13). Therefore, the present study hypothesized that NLRP2 may exhibit protective effects on vascular endothelial cells.

In the present study, the expression of NLRP2 in human umbilical vein endothelial cells (HUVECs) was knocked-down 
to investigate its functions in HUVEC proliferation, apoptosis, cell cycle and motility. By this, it was hoped to identify the function of NLRP2 in HUVECs and elucidate the underlying signaling pathway.

\section{Materials and methods}

Cell culture and transfection. HUVECs were purchased from the Chinese Academy of Sciences cell bank (Shanghai, China) and cultured in RPMI-1640 culture medium (Hyclone, GE Healthcare Life Sciences, Logan, UT, USA) at $37^{\circ} \mathrm{C}$ in $5 \% \mathrm{CO}_{2}$. The medium additions were fetal bovine serum (FBS; Gibco; Thermo Fisher Scientific, Inc., Waltham, MA, USA) and $10 \%$ penicillin and streptomycin (Beijing Solarbio Science \& Technology Co., Ltd., Beijing, China) at 100 units and $0.1 \mathrm{mg} / \mathrm{ml}$, respectively. When the cells entered into the logarithmic growth phase, cells were digested to form a single-cell suspension and plated in 6-well plates. When the cell density reached $\sim 80 \%, 20 \mathrm{nM}$ short interfering siNLRP2 or a scrambled siRNA (siNC) was transfected into the cells using Lipofectamine ${ }^{\circledR} 2000$ (Thermo Fisher Scientific, Inc.) according to the manufacturer's protocols. The sequences of siRNAs were as follows: siNLRP2, 5'-CGUACAGAAGCUGCUUUCCGG AGU-3' and siNC, 5'-UUCUCCGAACGUGUCACGUTT-3'. Following transfection for $24 \mathrm{~h}$, the mRNA expression of NLRP2 was determined by reverse transcription-quantitative polymerase chain reaction (RT-qPCR).

$R T-q P C R$. Following transfection with siNLARP2 or siNC for $48 \mathrm{~h}$, total RNA from HUVECs was extracted using TRIzol reagent (Cwbio, Beijing, China). Then, $1 \mu \mathrm{g}$ total RNA was reverse transcribed to cDNA using a reverse transcription system (Promega Corporation, Madison, WI, USA). Subsequently, PCR was performed using GoTaq qPCR master mix (Promega Corporation) on a 7500 Real-Time PCR system (Applied Biosystems; Thermo Fisher Scientific, Inc.). In the $25 \mu \mathrm{l}$ reaction system, $300 \mathrm{nmol} / 1$ primers were used. Thermal cycling conditions were: $2 \mathrm{~min}$ at $50^{\circ} \mathrm{C}$ and $10 \mathrm{~min}$ at $95^{\circ} \mathrm{C}$, followed by 40 cycles of $15 \mathrm{sec}$ at $95^{\circ} \mathrm{C}$ and $1 \mathrm{~min}$ at $60^{\circ} \mathrm{C}$. The primers were as follows: Glycogen synthase kinase- $3 \beta$ left, 5'-GAATTGCTGCGATGCGACAT-3' and right, 5'-TCG AAGAGCTAGGCAGAGGT-3'; GAPDH forward, 5'-TGA CTTCAACAGCGACACCCA-3', reverse, 5'-CACCCTGTT GCTGTAGCCAAA-3'. The fold-change in the expression of each gene was calculated using the $2^{-\Delta \Delta \mathrm{Cq}}$ method (14).

Cell Counting kit-8 (CCK-8) proliferation assay. HUVECs were digested to prepare a single-cell suspension and plated into a 96-well plate at a density of $1-5 \times 10^{3} /$ well. siNLRP 2 or siNC was transfected into HUVECs using Lipofectamine ${ }^{\circledR} 2000$ (Thermo Fisher Scientific, Inc.) according to the manufacturer's protocols. Cell viability was measured every $24 \mathrm{~h}$. A total of $10 \mu \mathrm{l}$ of CCK-8 reagent was added to each well prior to the assay and incubated for $1.5 \mathrm{~h}$ in a $37^{\circ} \mathrm{C}$ incubator, and the optical density value at $450 \mathrm{~nm}$ was measured using a microplate reader to plot the proliferation curve.

Flow cytometry for apoptosis detection. Cell apoptosis was analyzed using an Annexin V-fluorescein isothiocyanate (FITC) Apoptosis Detection kit I (4A Biotech Co., Ltd., Beijing, China).
Briefly, following transfection with siNLRP2 or siNC for $48 \mathrm{~h}$, HUVECs were collected and centrifuged at $300 \mathrm{x} \mathrm{g,} 25^{\circ} \mathrm{C}$ for 5 min. Then, cells were resuspended in $4^{\circ} \mathrm{C}$ precooled PBS and centrifuged at $300 \mathrm{x} \mathrm{g}, 25^{\circ} \mathrm{C}$ for $5 \mathrm{~min}$. The supernatant was carefully removed. Cells were resuspended by adding $1 \mathrm{X}$ binding buffer and adjusting the cell density to $1-5 \times 10^{6} / \mathrm{ml}$. To $100 \mu \mathrm{l}$ of cell suspension in a $5 \mathrm{ml}$ flow tube, $5 \mu \mathrm{l}$ of Annexin V/FITC was added, mixed and incubated for $5 \mathrm{~min}$. Then, $10 \mu \mathrm{l}$ of propidium iodide (PI) and $400 \mu \mathrm{l}$ of PBS were added prior to analyzing by a flow cytometer (BD Biosciences, Franklin Lakes, NJ, USA). FlowJo software (version 7.6.1; FlowJo LLC, Ashland, OR, USA) was used for statistical analysis.

Flow cytometry for cell cycle analysis. Following transfection with siNLRP2 or siNC for $48 \mathrm{~h}$, HUVECs were collected and washed with PBS three times. Following fixation with $70 \%$ ethanol at $-20^{\circ} \mathrm{C}$ overnight, cells were incubated with RNAase $(0.1 \mathrm{mg} / \mathrm{ml})$ and PI $(0.02 \mathrm{mg} / \mathrm{ml})$ at $37^{\circ} \mathrm{C}$ for $30 \mathrm{~min}$. Flow cytometry was used to analyze cell proportion in $\mathrm{G}_{1}, \mathrm{~S}$ and $\mathrm{G}_{2} / \mathrm{M}$ phases. Flowjo software (version 7.6.1) was used for statistical analysis.

Wound healing assay. HUVECs were plated in 6-well plates and cultured to $100 \%$ confluence. A single-line scratch of $\sim 600-700 \mu \mathrm{m}$ was created using a p200 pipette tip and cell debris was removed by gentle washing with PBS. Then, the medium was replaced with fresh medium containing either siNLRP 2 or siNC. Cells were imaged at 0 and 48 h by using an inverted microscope (magnification, $\mathrm{x} 40$; Olympus Corporation, Tokyo, Japan), and the wound closure was evaluated using ImageJ software (version 1.46, National Institutes of Health, Bethesda, MD, USA).

Transwell invasion and migration assay. For the invasion assay, Matrigel (BD Biosciences) was added to Transwell inserts (Corning Incorporated, Corning, NY, USA) and solidified for 4-6 h in a $37^{\circ} \mathrm{C}$ incubator. Then, $500 \mu \mathrm{l}$ of serum-free medium was added to the bottom chamber to hydrate the polyethylene terephthalate (PET) membrane for $2 \mathrm{~h}$. HUVECs $\left(5 \times 10^{4}\right)$ transfected with siNLRP2 or siNC were plated in the top chamber in $200 \mu \mathrm{l}$ of serum-free medium and $500 \mu \mathrm{l}$ of medium containing $10 \%$ FBS was added to the bottom chamber. Following incubation for $48 \mathrm{~h}$, cells remaining on the top surface of the PET membrane were removed and invading cells were fixed with $4 \%$ paraformaldehyde for $30 \mathrm{~min}$, stained with $0.1 \%$ crystal violet for $20 \mathrm{~min}$, and washed with PBS at room temperature. Cell numbers were counted in 5 random fields under an inverted microscope (magnification, x100). The migration assay procedure was similar to the invasion experiment except that no Matrigel was used.

Western blot analysis. HUVECs having been transfected for $48 \mathrm{~h}$ were lysed in radio immunoprecipitation assay buffer (Beyotime Institute of Biotechnology, Jiangsu, China) supplemented with $1 \%$ protease cocktail inhibitor I (Calbiochem; Merck KGaA, Darmstadt, Germany). The protein concentration was measured using a BCA Protein Assay kit (Pierce; Thermo Fisher Scientific, Inc.); $20 \mu \mathrm{g}$ of proteins were analyzed by $12 \%$ SDS-PAGE and transferred onto PVDF membranes. Subsequently, the membrane underwent blocking 
A

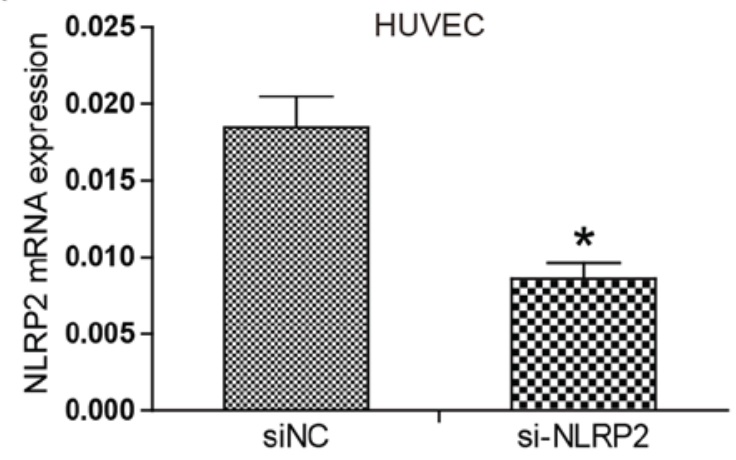

B

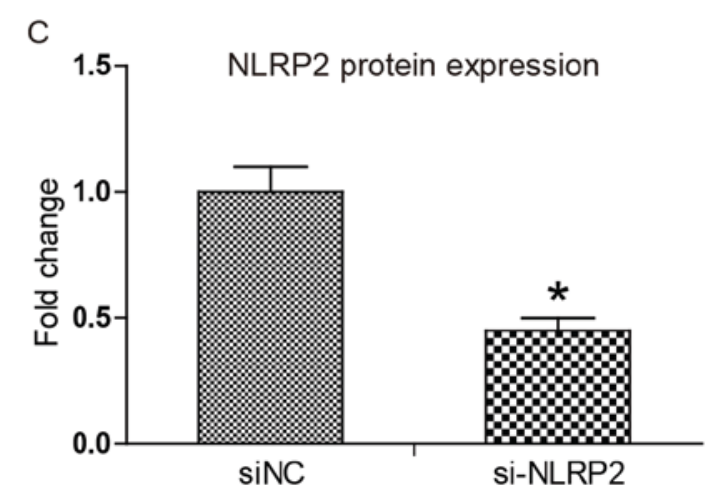

Figure 1. NLRP2 downregulation significantly reduced NLRP2 expression in HUVECs. (A) qPCR analysis demonstrated that NLRP2 mRNA level of HUVECs transfected with siNLRP2 was significantly reduced compared with siNC. (B and C) Western blot analysis demonstrated that NLRP2 protein level of HUVECs transfected with siNLRP2 was significantly reduced, compared with siNC. ${ }^{*} \mathrm{P}<0.05$ vs. siNC. NLRP2, nucleotide-binding oligomerization domain-like receptor protein 2; HUVECs, human umbilical vein endothelial cells; qPCR, quantitative polymerase chain reaction; siNC, scrambled short interfering RNA; siNLRP2, short interfering NLRP2.

in nonfat milk for $1.5 \mathrm{~h}$ at room temperature, was probed with primary antibodies at $4^{\circ} \mathrm{C}$ overnight and incubated with anti-rabbit or anti-mouse secondary antibodies for $1 \mathrm{~h}$. Finally, the protein bands were visualized by using a Pierce ECL Western Blotting Substrate (Pierce; Thermo Fisher Scientific, Inc.) and the density was quantified using ImageJ software (version 1.46). The primary antibodies used were as follows: Anti-NLRP2 (cat. no. SAB3500325, 1:1,000), anti-GAPDH (cat. no. G9545, 1:10,000; both Sigma-Aldrich; Merck KGaA), anti-caspase-3 (cat. no. ab13847, 1:1,000), anti-p53 (cat. no. ab26; 1:1,000), anti-Bcl-2-like protein 4 (Bax; cat. no. ab32503; 1:1,000), anti-B-cell lymphoma 2 (Bcl-2; cat. no. ab32124; 1:1,000), anti-p70S6 kinase (p70S6K; cat. no. ab176651, 1:1,000), anti-cyclin-dependent kinase 4 (CDK4; cat. no. ab108357, 1:1,000), anti-cyclinD1 (cat. no. ab134175. 1:1,000), anti-p-extracellular signal-regulated kinase (ERK; cat. no. ab192591, 1:1,000), anti-ERK (cat. no. ab224313, 1:1,000), anti-Raf (cat. no. ab137435; 1:1,000; all Abcam, Cambridge, UK). Horseradish peroxidase-conjugated goat anti-mouse or goat anti-rabbit IgG antibody (cat. no. A0192 and A0208, 1:1,000, Beyotime Institute of Biotechnology) was used as secondary antibodies.

Statistical analysis. Each experiment was performed at least three times. All data analyses were conducted by using SPSS 17.0 software (SPSS Inc., Chicago, IL, USA). The results were manifested as mean \pm standard deviation. All the comparisons were performed using unpaired student t-test. $\mathrm{P}<0.05$ was considered to indicate a statistically significant difference.

\section{Results}

Inhibition of HUVEC proliferation by knocking down NLRP2 expression. To investigate the action of NLRP2 in HUVECs, NLRP2 expression was knocked-down using RNA interference technology (siNLRP2), along with a non-targeting scrambled siRNA (siNC) that was constructed to use as a negative control in all assays. The interference efficiency of siNLRP2 on NLRP2 expression was examined via mRNA levels using RT-qPCR. Fig. 1A demonstrates that siNLRP2 exerted high efficiency in knocking down NLRP2 mRNA expression compared with the siNC, with an inhibition rate reaching 54\%. Western blotting results in Fig. 1B and $\mathrm{C}$ indicate that protein expression of NLRP2 was also significantly reduced in the siNLRP2 group compared with the siNC group $(\mathrm{P}<0.05)$. The results of the in vitro $\mathrm{CCK}-8$ proliferation assay indicated that the cell viability of siNLRP2-transfected HUVECs was notably decreased in comparison with siNC-transfected cells $(\mathrm{P}<0.05$; Fig. 2A). Observation of HUVEC morphology (Fig. 2B) suggested that transfection of siNLRP2 led to apoptosis-like changes including cell shrinkage and the formation of vacuoles.

Enhancement of apoptosis by knocking down NLRP2 expression. Flow cytometry was performed to assess apoptosis of HUVECs. Measurement was based on staining with Annexin V-FITC and PI. The resulting plot, presented in Fig. 3A, is divided into 4 quadrants. Early apoptotic cells populating the lower right quadrant are stained only by AnnexinV-FITC. Late apoptotic cells populating the upper right quadrant are stained by Annexin V-FITC 

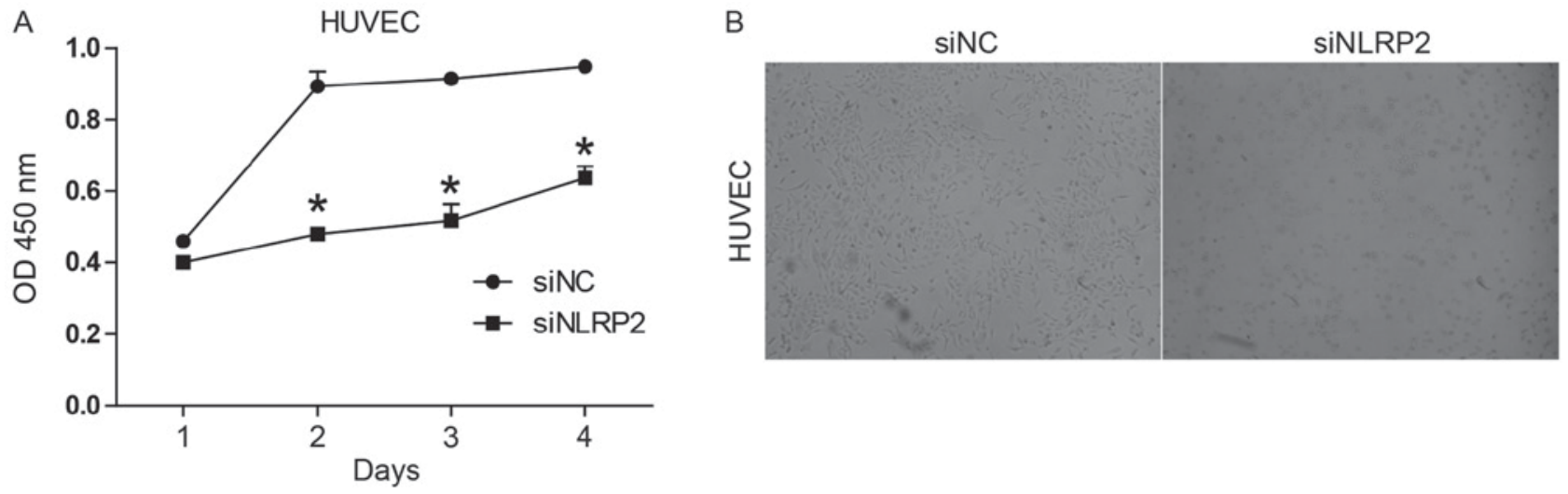

Figure 2. NLRP2 downregulation significantly inhibited cell proliferation in HUVECs and led to apoptosis-like morphology. (A) HUVEC proliferation curve when transfected with siNC or siNLRP2. (B) HUVEC morphology when transfected with siNC or siNLRP2. Scale bar, $500 \mu \mathrm{m}$. Magnification, x40. ${ }^{*} \mathrm{P}<0.05$ vs. siNC. NLRP2, nucleotide-binding oligomerization domain-like receptor protein 2; HUVECs, human umbilical vein endothelial cells; siNC, scrambled short interfering RNA; siNLRP2, short interfering NLRP2; OD, optical density.

A

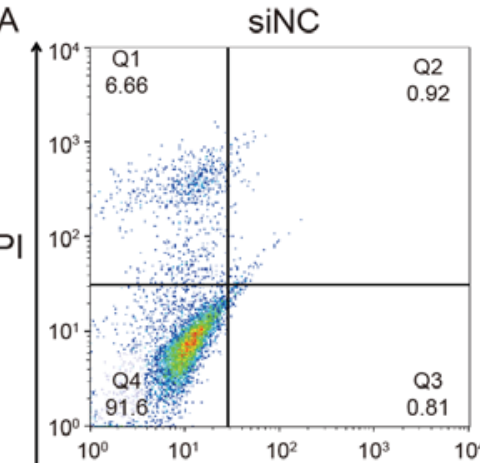

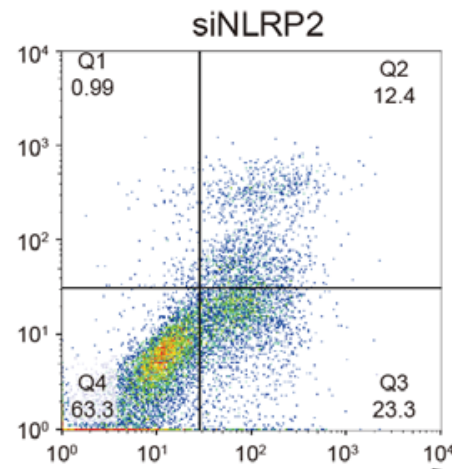

Annexin V
B

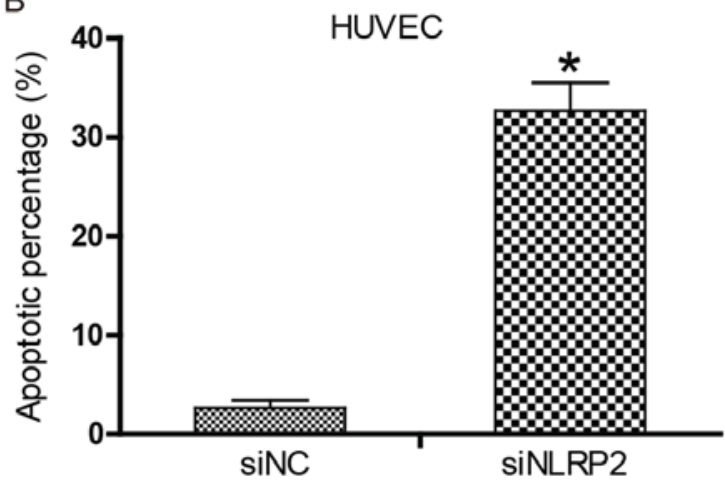

D
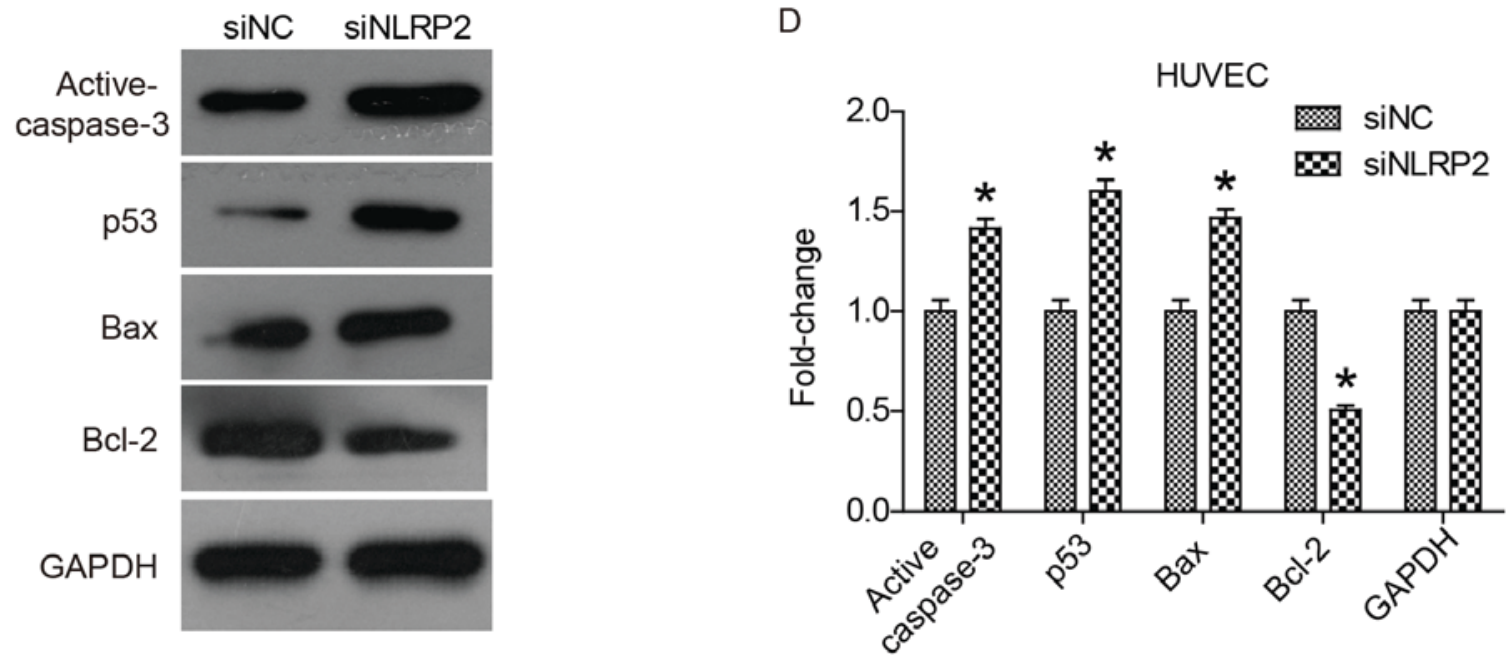

Figure 3. NLRP2 downregulation induced cell apoptosis in HUVECs and changed apoptosis-associated gene expression. (A and B) Flow cytometry demonstrated that apoptosis of HUVECs was significantly enhanced when transfected with siNLRP2. (C and D) western blot analysis demonstrated that the expression of the apoptosis-associated genes active-caspase 3, p53 and Bax were upregulated while Bcl- 2 was downregulated. " $\mathrm{P}<0.05$ vs. siNC. NLRP2, nucleotide-binding oligomerization domain-like receptor protein 2; HUVECs, human umbilical vein endothelial cells; Bax, Bcl-2-like protein 4; Bcl-2, B-cell lymphoma 2 siNC, scrambled short interfering RNA; siNLRP2, short interfering NLRP2; PI, propidium iodide.

and PI. This suggested that siNLRP2 significantly promoted early and late apoptosis of HUVECs compared with siNC $(\mathrm{P}<0.05)$. The total apoptotic proportion increased from 1.73 to $35.7 \%$ ( $\mathrm{P}<0.05$; Fig. 3A and B). To clarify the pro-apoptosis mechanism of siNLRP2, the expression of apoptosis-associated genes was investigated. The results suggested that the expression of the pro-apoptotic genes p53, Bax and active-caspase 3 was significantly increased and the expression of the anti-apoptotic gene $\mathrm{Bcl}-2$ was significantly decreased in the siNLRP2 group $(\mathrm{P}<0.05$; Fig. $3 \mathrm{C}$ and $\mathrm{D})$. These gene expression changes would 


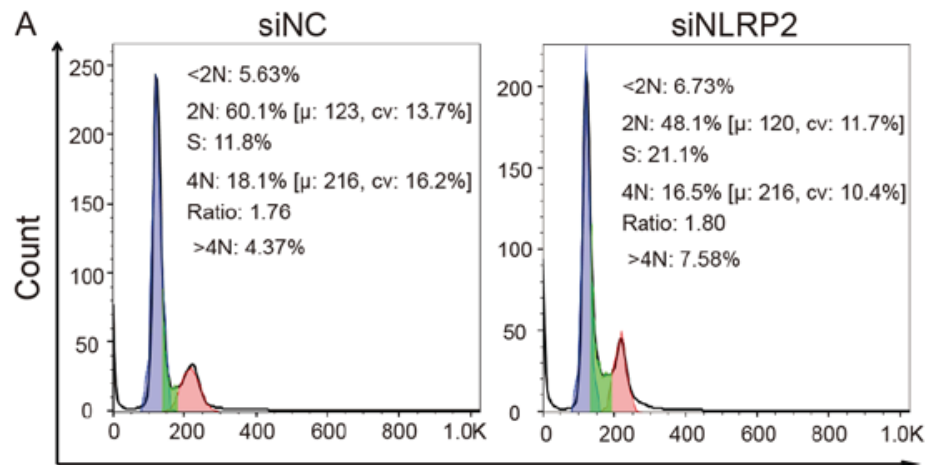

DNA content

C

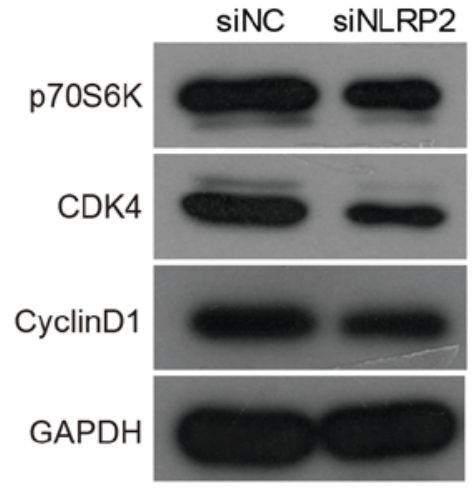

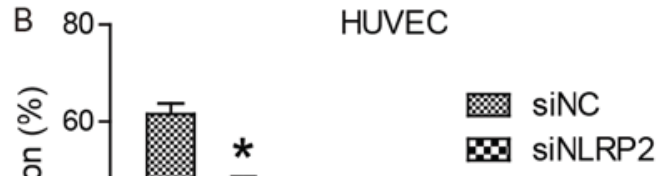

$\mathbf{\infty}$ siNLRP2
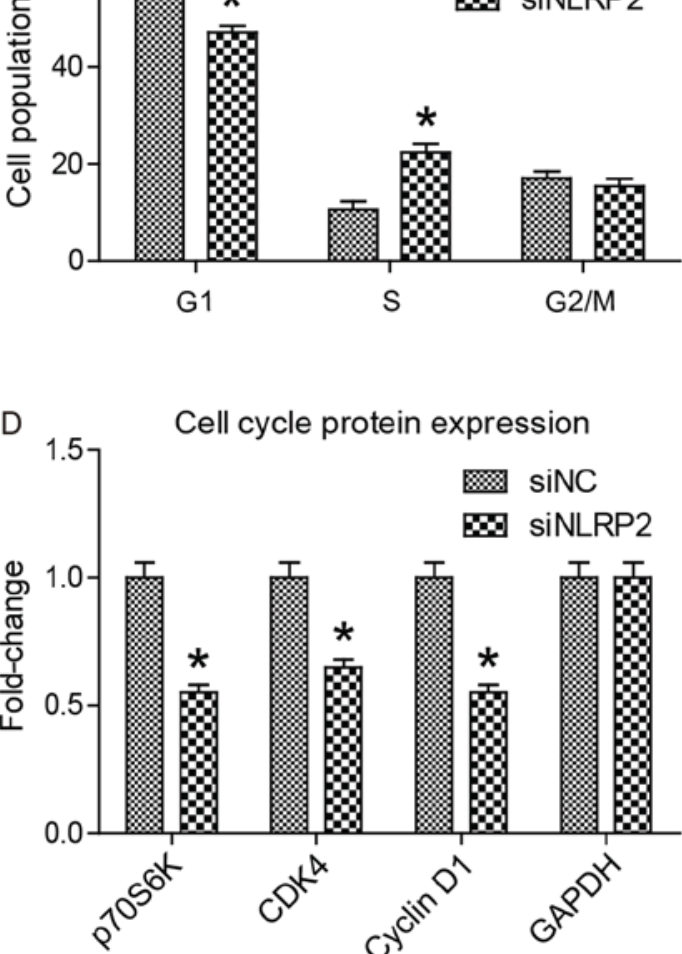

Figure 4. NLRP2 downregulation induced cell cycle arrest and changed cell cycle-associated gene expression. (A and B) Flow cytometry demonstrated that the HUVEC cell cycle was arrested when transfected with siNLRP2. (C and D) western blot analysis demonstrated that the expression of cell cycle-associated genes P70S6K, CDK4, and cyclinD1 were downregulated when transfected with siNLRP2. $\mathrm{P}<0.05$ vs. siNC. NLRP2, nucleotide-binding oligomerization domain-like receptor protein 2; siNLRP2, short interfering NLRP2; P70S6K, p70S6 kinase; CDK4, cyclin-dependent kinase 4.

cause cytochrome $c$ release and mitochondria-dependent cell apoptosis. These results suggested that knockdown of NLRP2 with siNLRP2 promoted HUVEC cell apoptosis by regulating apoptosis-associated gene expression.

Cell cycle arrest by knocking down NLRP2 expression. To determine if knockdown of NLRP2 expression interfered with cell cycle progression, a cell cycle analysis was performed. The principle of this assay is based on alterations in DNA content throughout the cell cycle progression. DNA content was detected by PI staining and flow cytometry. The data in Fig. 4A and B demonstrated that siNLRP2 transfection significantly increased the proportion of S-phase cells (from 11.8 to $21.1 \%$; $\mathrm{P}<0.05$ ), but the proportion of $\mathrm{G}_{1}$-phase cells dropped from $60.1-48.1 \%$. This suggested that knockdown of NLRP 2 could arrest cell cycle of HUVECs at $\mathrm{S}$ phase. To explain the underlying mechanism, a western blot analysis was performed to determine alterations in the expression of cell cycle-associated genes including p70S6K, CDK4, cyclin D1 and p53. The results, presented in Fig. $4 \mathrm{C}$ and D, indicated that the expression of cell cycle driving genes including p70S6K, CDK4 and cyclin D1 was significantly decreased $(\mathrm{P}<0.05)$. Overall, the results suggested that knockdown of NLRP2 halted cell cycle progression at the $\mathrm{S}$ phase by regulating cell cycle-associated gene expression.

Inhibition of cell migration and invasion by knocking down NLRP2 expression. To determine if siNLRP2 transfection had an effect on cell migration, a scratch assay was performed. Wound closure was examined at 0 and $48 \mathrm{~h}$ following wounding (Fig. 5A and B). No significant difference was observed between siNC and siNLRP2 at $0 \mathrm{~h}$. Following transfection for $48 \mathrm{~h}$, siNLRP2 led to significantly decreased wound closure compared with the siNC group $(\mathrm{P}<0.05)$. Since migration and proliferation affect wound closure in the wound healing assay, a Transwell migration assay was performed to specifically evaluate cell migration. The results are summarized in Fig. 5C and D. Regarding the migrated cell numbers, cell migration was reduced 4 -fold as a result of transfection with siNLRP2 compared with siNC. The effect of siNLRP2 transfection on HUVEC cell invasion was tested by using a Transwell invasion assay. The data (Fig. 5E and F) demonstrated that the number of HUVECs that invaded the Matrigel was significantly reduced in the siNLRP2 group compared with siNC group $(\mathrm{P}<0.05)$. Statistical analysis indicated that the inhibitory effect of siNLRP2 on cell invasion was $76 \%$ of that of siNC. Together, these results demonstrated that knockdown of NLRP2 expression markedly inhibited HUVEC migration and invasion.

Knocking down NLRP2 inhibits the MAPK signaling pathway. The MAPK signaling pathway is an important signaling pathway that regulates cell proliferation, apoptosis and migration (15). Among the signaling components, MAPK, ERK and Raf serve a central regulating role, and their abnormal activation, 
A

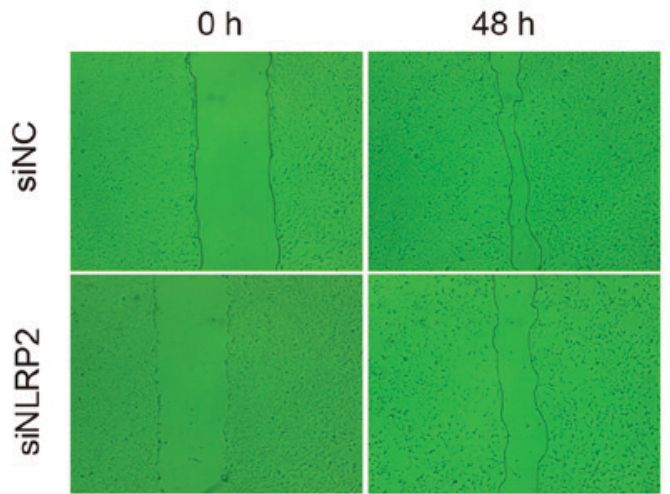

C

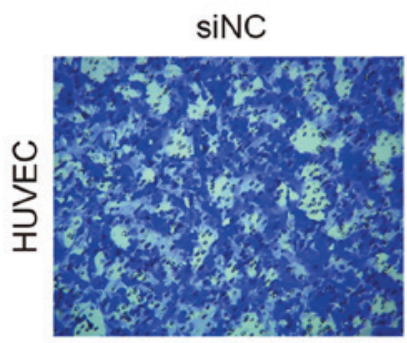

$\mathrm{E}$

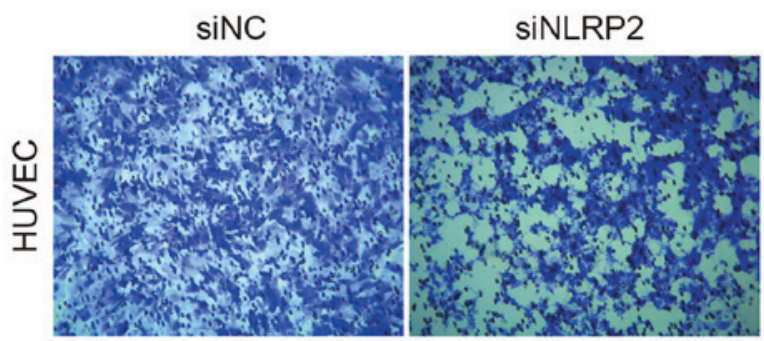

B

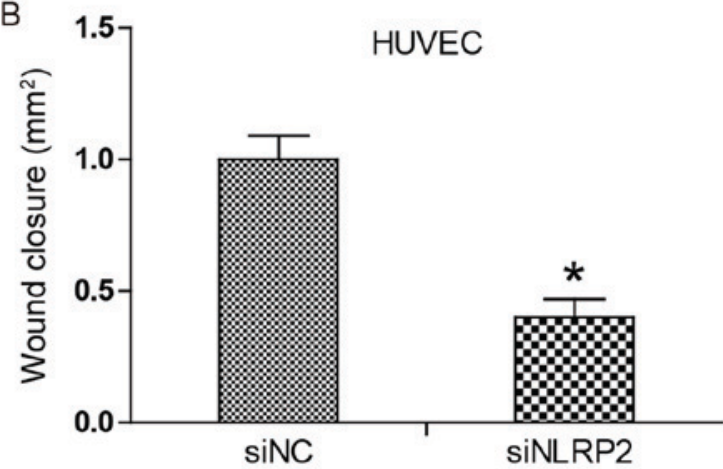

D

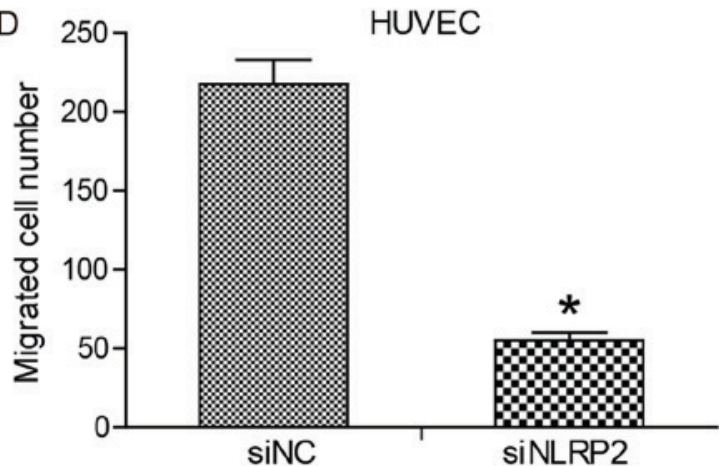

$\mathrm{F}$

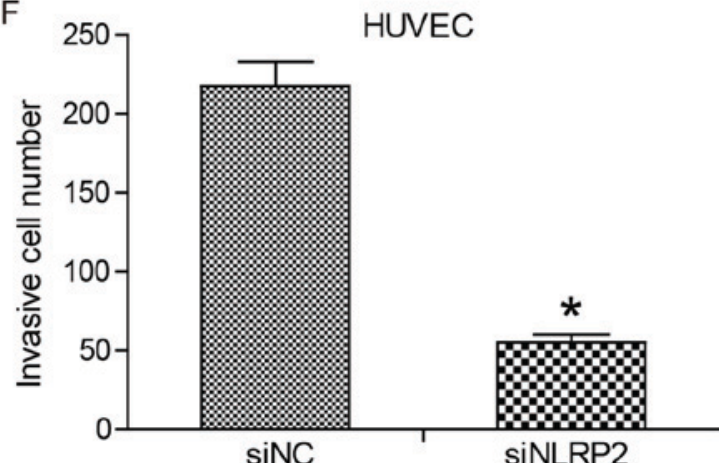

Figure 5. NLRP2 downregulation significantly inhibited cell migration and invasion in HUVECs. (A and B) wound healing assay (magnification, x40) and (C and D) Transwell migration assay (magnification, x100) demonstrated that siNLRP2 transfection significantly inhibited cell migration in HUVECs. (E) and (F) Transwell invasion assay (magnification, x100) demonstrated that siNLRP2 transfection significantly inhibited cell invasion in HUVECs. "P<0.05 vs. siNC. NLRP2, nucleotide-binding oligomerization domain-like receptor protein 2; HUVECs, human umbilical vein endothelial cells; siNLRP2, short interfering NLRP2.
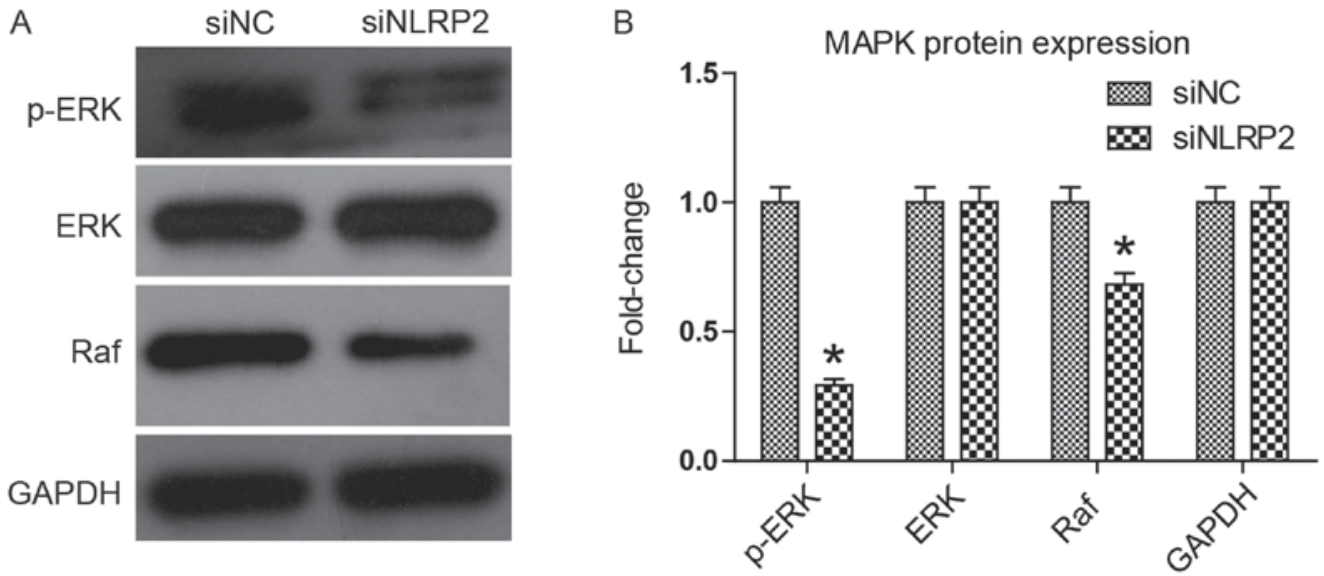

Figure 6. NLRP2 downregulation inhibited the MAPK signaling pathway. (A and B) p-ERK and Raf were key regulating protein in MAPK signaling pathway, whose downregulation represented inhibition of the pathway. "P<0.05 vs. siNC. NLRP2, nucleotide-binding oligomerization domain-like receptor protein 2; MAPK, mitogen-activated protein kinase; p-, phosphorylated; ERK, extracellular signal-regulated kinase. 
expression or upregulation frequently causes an uncontrolled proliferation as in cancer cells $(16,17)$. The present study investigated the effect of siNLRP2 transfection on the MAPK signaling pathway by using western blot analysis. The results in Fig. 6 indicated that siNLRP2 transfection significantly downregulated Raf expression and ERK phosphorylation $(\mathrm{P}<0.05)$, while no evident change was identified in ERK expression. This suggests that knocking down NLRP2 inhibited the MAPK signaling pathway, which explains the above inhibitory activity of siNLRP2 on HUVECs.

\section{Discussion}

NLRs, hosted by the innate immune system, are mainly involved in recognizing harmful endogenous and exogenous molecular patterns and forming inflammasomes $(5,18)$. NLRP2 belongs to a group of NLRPs, which is a subfamily of NLRs. In addition to mediating the activation of inflammasomes in innate immune responses (19), NLRP2 has been mainly described as participating in reproduction and embryonic development $(8,10,20,21)$. Other studies have revealed a correlation with ischemic stroke, bipolar disorder and sibling allogeneic stem cell transplantation $(13,19,22)$. However, NLRP2 expression and whether it serves an important physiological function in vascular endothelial cells has yet to be elucidated.

The present study used HUVECs as a model to investigate NLRP2 expression and function in vascular endothelial cells. siNLRP2 was constructed to knock down the expression of NLRP2 and a scrambled siRNA was used as a negative control. qPCR and western blot analysis indicated that NLRP2 was basally expressed in HUVECs and that siNLRP2 exerted effective interference. Using a CCK- 8 assay, it was identified that siNLRP2 significantly inhibited the proliferation of HUVECs and led to apoptosis-like morphological changes, including cell shrinkage and blebbing. The motility of cells, including migration and invasion, was also identified to be suppressed by siNLRP 2 in a wound healing and in a Transwell assay. An apoptosis and cell cycle detection assay revealed that siNLRP2 induced apoptosis and inhibited HUVEC cell cycle progression. In addition, apoptosis-associated gene expression was also altered in a manner consistent with apoptosis activation, including increased p53 and active-caspase 3 expression, and a decreased $\mathrm{Bcl}-2 / \mathrm{Bax}$ ratio. With respect to cell cycle-associated genes, p70S6K, CDK4 and cyclin D1 were downregulated, and conversely, p53 was upregulated. p53 was responsible for preventing cell cycle progression and inducing apoptosis when cells were in abnormal states. Additionally, members of the MAPK signaling pathway, ERK and Raf, were also investigated. The results suggested that ERK phosphorylation and Raf expression were elevated, while ERK expression was unchanged. The results indicated that downregulation of NLPR2 led to the inhibition of the MAPK signaling pathway. MAPK cascade activation is the center of a variety of signaling pathways and serves a key role in cell proliferation and differentiation (23-26).

In conclusion, for the first time, to the best of the authors' knowledge, NLRP2 function in HUVECs has been investigated. The results suggested that NLRP2 serves an important role in maintaining HUVEC viability and motility via the
MAPK signaling pathway. In combination with a previous study demonstrating that NLRP2 expression is significantly enhanced in a mouse model of ischemic stroke (13), it was assumed that NLRP2 may exert a vessel protection function in ischemic stroke. These results also provide a novel therapeutic strategy for tumor angiogenesis. Gene therapy or monoclonal antibodies may be used to selectively inhibit NLRP2 function in tumors and suppress tumor angiogenesis. Altogether, the findings of the present study open a new field of NLRP2 functional research that could help to find novel treatment strategies for vascular endothelial-associated diseases.

\section{Acknowledgements}

Not applicable.

\section{Funding}

No funding was received.

\section{Availability of data and materials}

Not applicable.

\section{Authors' contributions}

$\mathrm{XZ}$ and $\mathrm{XL}$ performed the experiments and wrote the manuscript; YG and LY made substantial contributions to the acquisition of data. FQ made substantial contributions to the conception and design of the present study and has given final approval of the version to be published.

\section{Ethics approval and consent to participate}

Not applicable.

\section{Patient consent for publication}

Not applicable.

\section{Competing interests}

The authors declare that they have no competing interests.

\section{References}

1. Medzhitov R: Origin and physiological roles of inflammation. Nature 454: 428-435, 2008

2. Maslanik T, Mahaffey L, Tannura K, Beninson L, Greenwood BN and Fleshner M: The inflammasome and danger associated molecular patterns (DAMPs) are implicated in cytokine and chemokine responses following stressor exposure. Brain Behav Immun 28: 54-62, 2013.

3. Bauer RN, Diaz-Sanchez D and Jaspers I: Effects of air pollutants on innate immunity: The role of Toll-like receptors and nucleotide-binding oligomerization domain-like receptors. J Allergy Clin Immunol 129: 14-26, 2012.

4. Kim YK, Shin JS and Nahm MH: NOD-like receptors in infection, immunity, and diseases. Yonsei Med J 57: 5-14, 2016.

5. Abderrazak A, Syrovets T, Couchie D, El Hadri K, Friguet B, Simmet T and Rouis M: NLRP3 inflammasome: From a danger signal sensor to a regulatory node of oxidative stress and inflammatory diseases. Redox Biol 4: 296-307, 2015. 
6. Elinav E, Strowig T, Kau AL, Henao-Mejia J, Thaiss CA, Booth CJ, Peaper DR, Bertin J, Eisenbarth SC, Gordon JI and Flavell RA: NLRP6 inflammasome regulates colonic microbial ecology and risk for colitis. Cell 145: 745-757, 2011.

7. Brickler T, Gresham K, Meza A, Coutermarsh-Ott S, Williams TM, Rothschild DE, Allen IC and Theus MH: Nonessential role for the NLRP1 inflammasome complex in a murine model of traumatic brain injury. Mediators Inflamm 2016 6373506, 2016

8. Tilburgs T, Meissner TB, Ferreira LMR, Mulder A, Musunuru K, Ye $\mathrm{J}$ and Strominger JL: NLRP2 is a suppressor of NF- $\kappa \mathrm{B}$ signaling and HLA-C expression in human trophoblasts $\uparrow,+$. Biol Reprod 96: 831-842, 2017.

9. Mahadevan S, Sathappan V, Utama B, Lorenzo I, Kaskar K and Van den Veyver IB: Maternally expressed NLRP2 links the subcortical maternal complex (SCMC) to fertility, embryogenesis and epigenetic reprogramming. Sci Rep 7: 44667, 2017.

10. Peng H, Chang B, Lu C, Su J, Wu Y, Lv P, Wang Y, Liu J, Zhang B, Quan F, et al: Nlrp2, a maternal effect gene required for early embryonic development in the mouse. PLoS One 7: e30344, 2012.

11. Aird WC: Phenotypic heterogeneity of the endothelium: I. Structure, function, and mechanisms. Circ Res 100: 158-173, 2007.

12. Kinnunen K, Piippo N, Loukovaara S, Hytti M, Kaarniranta K and Kauppinen A: Lysosomal destabilization activates the NLRP3 inflammasome in human umbilical vein endothelial cells (HUVECs). J Cell Commun Signal 11: 275-279, 2017.

13. Sun X, Song X, Zhang L, Sun J, Wei X, Meng L and An J: NLRP2 is highly expressed in a mouse model of ischemic stroke. Biochem Biophys Res Commun 479: 656-662, 2016.

14. Livak KJ and Schmittgen TD: Analysis of relative gene expression data using real-time quantitative PCR and the 2(-Delta Delta C(T)) method. Methods 25: 402-408, 2001

15. Santarpia L, Lippman SM and El-Naggar AK: Targeting the MAPK-RAS-RAF signaling pathway in cancer therapy. Expert Opin Ther Targets 16: 103-119, 2012.

16. Maurer G, Tarkowski B and Baccarini M: Raf kinases in cancer-roles and therapeutic opportunities. Oncogene 30: 3477-3488, 2011

17. McCubrey JA, Steelman LS, Chappell WH, Abrams SL, Wong EW, Chang F, Lehmann B, Terrian DM, Milella M, Tafuri A, et al: Roles of the Raf/MEK/ERK pathway in cell growth, malignant transformation and drug resistance. Biochim Biophys Acta 1773: 1263-1284, 2007.
18. Ozaki E, Campbell M and Doyle SL: Targeting the NLRP3 inflammasome in chronic inflammatory diseases: Current perspectives. J Inflamm Res 8: 15-27, 2015.

19. Vizlin-Hodzic D, Zhai Q, Illes S, Södersten K, Truvé K, Parris TZ, Sobhan PK, Salmela S, Kosalai ST, Kanduri C, et al: Early onset of inflammation during ontogeny of bipolar disorder: The NLRP2 inflammasome gene distinctly differentiates between patients and healthy controls in the transition between iPS cell and neural stem cell stages. Transl Psychiatry 7: e1010, 2017.

20. Fontalba A, Gutierrez O and Fernandez-Luna JL: NLRP2, an inhibitor of the NF-kappaB pathway, is transcriptionally activated by NF-kappaB and exhibits a nonfunctional allelic variant. J Immunol 179: 8519-8524, 2007.

21. Aghajanova L, Mahadevan S, Altmäe S, Stavreus-Evers A, Regan L, Sebire N, Dixon P, Fisher RA and Van den Veyver IB: No evidence for mutations in NLRP7, NLRP2 or KHDC3L in women with unexplained recurrent pregnancy loss or infertility. Hum Reprod 30: 232-238, 2015.

22. Granell M, Urbano-Ispizua A, Pons A, Aróstegui JI, Gel B, Navarro A, Jansa S, Artells R, Gaya A, Talarn C, et al: Common variants in NLRP2 and NLRP3 genes are strong prognostic factors for the outcome of HLA-identical sibling allogeneic stem cell transplantation. Blood 112: 4337-4342, 2008.

23. Fragale A, Tartaglia M, Wu J and Gelb BD: Noonan syndrome-associated SHP2/PTPN11 mutants cause EGF-dependent prolonged GAB1 binding and sustained ERK2/MAPK1 activation. Hum Mutat 23: 267-277, 2004.

24. Apáti A, Jánossy J, Brózik A, Bauer PI and Magócsi M: Calcium induces cell survival and proliferation through the activation of the MAPK pathway in a human hormone-dependent leukemia cell line, TF-1. J Biol Chem 278: 9235-9243, 2003.

25. Carter BZ, Mak DH, Schober WD, Cabreira-Hansen M, Beran M, McQueen T, Chen W and Andreeff M: Regulation of survivin expression through Bcr-Abl/MAPK cascade: Targeting survivin overcomes imatinib resistance and increases imatinib sensitivity in imatinib-responsive CML cells. Blood 107: 1555-1563, 2006.

26. Schmid RS, Graff RD, Schaller MD, Chen S, Schachner M, Hemperly JJ and Maness PF: NCAM stimulates the Ras-MAPK pathway and CREB phosphorylation in neuronal cells. J Neurobiol 38: 542-558, 1999.

This work is licensed under a Creative Commons Attribution-NonCommercial-NoDerivatives 4.0 International (CC BY-NC-ND 4.0) License. 